\title{
CONSIDERAZIONI INTRODUTTIVE
}

\section{Quanta erit infelicitas urbis illius, in qua virorum officia mulieres occupabunt $!^{1}$}

Ė Lattanzio a lamentare nella sua Epitome la cattiva sorte di una città in cui le donne avessero assunto i compiti pubblici e politici, tradizionalmente di esclusiva competenza maschile. La lunga storia di Roma dimostrava il carattere irrealistico di questa eventualità: il pudor, virtù vincolante per la condotta delle matrone secondo il mos maiorum, le costringeva, infatti, in una dimensione prevalentemente privata, ma soprattutto le norme che presiedevano alla vita istituzionale, militare, civica precludevano l'attività pubblica alla componente femminile della società.

Tale estraneità delle donne rispetto alla vita cittadina subì, tuttavia, una significativa, anche se per molti aspetti solo temporanea, trasformazione tra la fine del II e il I secolo a.C., quando si crearono condizioni tanto particolari da permettere alle matrone di violare i confini fisici ma anche 'ideologici' rappresentati dalla domus, solo spazio e ambito pertinente all'azione femminile, e di agere in re publica, interferendo nella vita pubblica e politica dell'Urbe. In questi decenni significativamente coincidenti con la vita di Cicerone, identificato dalla critica come il probabile ispiratore della riflessione di Lattanzio, la successione ininterrotta di scontri intestini determinò infatti l'allontanamento di molti uomini dalle sedi della decisione politica e un sovvertimento delle regole della vita civica, che aprirono ad alcune donne - mogli, madri, figlie e sorelle dei detentori del potere -, spazi di azione prima loro preclusi. Se nella maggioranza dei casi questi comportamenti contrari alla tradizione determinarono la delegittimazione delle loro promotrici, in altre evenienze il vincolo della pietas nei confronti di quei familiari che con il loro operato esse tentavano di tutelare, consentì di giustificare le infrazioni della tradizione di cui le matrone si resero responsabili, preservando la loro immagine sia nella valutazione dei contemporanei sia nella memoria dei posteri. E ciò permise che nel percorso di normalizzazione avviato con la ritrovata pax augustea, pur in una tendenza evidente a ricondurre i comportamenti femminili nell'alveo della tradizione, persistessero alcune delle trasformazioni che nel ruolo pubblico e politico femminile si erano prodotte nel corso della tarda repubblica.

Lo spettro cronologico che connota questa raccolta di saggi, le cui riflessioni si dispiegano attraverso un arco temporale che dal II secolo a.C. si estende fino al I d.C., risponde proprio alla duplice finalità di questo volume: da un lato, attraverso un approccio sincronico si sono intesi definire, nel momento del loro prodursi, i molti aspetti del complesso fenomeno che nella tarda repubblica consentì l'attività delle matrone sulla scena pubblica; dall'altro, in prospettiva diacronica, ci si è proposti di verificare le forme della condizione femminile che si determinò allorché, dopo decen-

\footnotetext{
${ }^{1}$ Lact. Epit. 33 (38) 5.
} 
ni di trasformazione, si raggiunsero nel I secolo d.C. nuovi equilibri politici e sociali in bilico tra innovazione e conservazione.

Con un'attenzione specifica alla tarda repubblica, si è perseguito quindi in prima istanza l'obiettivo di ricostruire il profilo delle protagoniste della trasformazione, le sedi e le occasioni della loro azione, le motivazioni e le modalità del loro operare in domo e in re publica. In una prospettiva di ampia durata, si è individuata la seconda finalità nel delineare l'entità e le forme della persistenza, oltre il periodo della crisi, delle caratteristiche dell'iniziativa pubblica e politica matronale, con attenzione alla costruzione in rebus del modello femminile ma anche alla sua incidenza condizionante nella memoria post res degli eventi.

I contributi raccolti in questo volume sono in larga parte la rielaborazione per iscritto, anche alla luce della discussione, delle relazioni tenute in occasione del Convegno Internazionale "Matronae in domo et in re publica agentes. Spazi e occasioni dell'azione femminile nel mondo romano fra tarda repubblica e primo impero", che ha avuto luogo a Venezia il 16 e il 17 ottobre 2014. L'occasione congressuale ha rappresentato il momento conclusivo del Progetto finanziato dall'Università Ca' Foscari nel 2012 dal titolo "Matrone in politica e politica delle matrone. Spazi e modalità dell'azione femminile nella tarda repubblica romana", scaturito dalla collaborazione tra studiosi italiani e francesi, specialisti di diversi ambiti disciplinari: Francesca Cenerini (Università Alma Mater Studiorum di Bologna), Alfredo Buonopane (Università degli Studi di Verona), Maria Letizia Caldelli (Sapienza Università di Roma), François Chausson (Université Paris I Panthéon Sorbonne), Isabelle Cogitore (Université Grenoble III Stendhal), Gian Luca Gregori (Sapienza Università di Roma), Anna Lina Morelli (Università Alma Mater Studiorum di Bologna), Luigi Sperti e Francesca Rohr (Università Ca' Foscari di Venezia), coordinatore della ricerca. I contributi presentati in sede congressuale sono affiancati in questo volume da saggi incentrati su episodi e personaggi, che si rivelano decisivi per la comprensione dell'azione matronale in domo et in republica tra II secolo a.C. e I secolo d.C.

Convegno, progetto e pubblicazione di questo volume rientrano tra le attività del gruppo di ricerca internazionale GIEFFRA (Groupe International d'Etudes sur les Femmes et la Famille dans la Rome Antique), coordinato da Francesca Cenerini, François Chausson, Isabelle Cogitore e Francesca Rohr: esso si propone di costituire una sede di confronto scientifico sui temi della donna e della famiglia nel mondo romano tra studiosi di formazione, competenze e nazionalità diverse attraverso l'ineludibile esegesi della documentazione antica.

L'organizzazione del Convegno e la pubblicazione di questo volume di Atti molto devono al patrocinio di importanti enti e gruppi di ricerca: l'Università Ca' Foscari di Venezia, che ha assicurato anche l'imprescindibile sostegno finanziario, l'Università Alma Mater Studiorum di Bologna, l'Associazione per lo sviluppo e la diffusione degli 
Studi sull'Italia romana Terra Italia, l'Associazione italiana di Cultura Classica - delegazione di Venezia, il Gruppo internazionale di ricerca sulla donna e la famiglia in Roma antica GIEFFRA.

Il volume si pone in prosecuzione ideale del lavoro pionieristico svolto nei due Seminari sulla condizione femminile organizzati da Alfredo Buonopane e Francesca Cenerini nel 2002 e nel 2004; benché essi abbiano posto l'accento in termini privilegiati sul record epigrafico, questi Atti, i cui contributi si connotano per un approccio multidisciplinare, si presentano come Terzo Seminario sulla condizione femminile perché da tali esperienze hanno ereditato l'attenzione specifica per il soggetto femminile nel mondo romano, l'apertura internazionale e la volontà di un confronto ancorato alle fonti.

Francesca Cenerini

Francesca Rohr Vio 